\title{
A three-part bilateral swinglock design denture revisited
}

\author{
C. W. Barclay, ' M. D. Russell, ${ }^{2}$ and P. Murphy, ${ }^{3}$
}

\section{This case was first reported by one of the authors in 1987, subsequent to the patient being treated using a combination of a hinge and split pin sectional denture. The same patient attended for review some I 4 years later for assessment of this denture at which stage a replacement was made.}

$\mathrm{F}_{\mathrm{i}}^{\mathrm{i}}$ ixed bridgework or dental implants are often a common line of treatment when considering the restoration of a limited bounded saddle edentulous area. However desirable such treatment may be, local, general or economic factors often indicate the use of a removable prosthesis instead. Such an appliance however may have inherent limitations, in particular a unidirectional path of insertion which therefore limits the available undercuts that can be used for retention. ${ }^{1}$ This may be overcome in many cases by the design and construction of a sectional denture consisting of more than one part each with an individual path of insertion, the components being joined together by some mechanism once seated. There are two main methods of uniting component parts of a sectional denture; either a lock and bolt design ${ }^{2}$ or a split post design. ${ }^{3}$ Simmons described the use of a swinglock removable partial denture to allow utilisation of unfavourable undercuts. ${ }^{4}$ The prime benefit of the swinglock is its ability to use a buccal or labial connector and so make use of the natural undercut in the anterior region of the mouth.

In spite of the basic designs already referred to there remain mouths which,

\footnotetext{
${ }^{1}$ Lecturer/Honorary Consultant in Restorative Dentistry, School of Dentistry, The University of Birmingham, St Chad's Queensway, Birmingham B4 6NN; ${ }^{2}$ Consultant in Restorative Dentistry, School of Dentistry, The Queens University of Belfast, Royal Victoria Hospital, Grosvenor Road, Belfast BT12 6BA; ${ }^{3}$ Senior Prosthetic Technician, School of Dentistry, The University of Birmingham, St Chad's Queensway, Birmingham B4 6NN

${ }^{*}$ Correspondence to: C. W. Barclay email:c.w.barclay@bham.ac.uk

REFEREED PAPER

Received 18.08.00; Accepted 23.11.00

(C) British Dental Journal 2001; 190: 538-540
}

because of malposition of teeth or anatomical form, present challenges to the prosthodontist who must consider increasing the complexity of design to produce a successful clinical result.

One such case has been reported ${ }^{5}$ and it is the purpose of this short paper to review the success of the technique over a period of 14 years and plan for the future.

\section{Case report}

A 72-year-old woman was referred by her GDP regarding maintenance and possible replacement of a three-part sectional denture which had been constructed some 14 years previously. ${ }^{5}$ The records indicated that she had been referred initially in early 1983 for the provision of bridgework to replace the missing upper central incisors which had been lost as a result of trauma. After clinical assessment and the preparation of study casts it was decided to construct a cobalt

\section{In brief}

- The management of an edentulous anterior bounded saddle in a situation where bridgework was not indicated and conventional removable dentures would not be possible.

- The benefits of buccal connectors in a situation where a deep complete overbite precluded the use of palatal connectors.

- The complexity of a removable prosthesis in itself will not be the sole determinant on longevity of service.

- The use of sectional and swinglock dentures provide a useful alternative in the management of edentulous spaces. chromium partial denture. Various designs were considered and finally a three-part bilateral swinglock denture was fitted several months later. The patient initially had some discomfort around the anterior section subsequent to insertion but after adjustments the denture had been satisfactory.

Since the initial denture had been constructed, no additional or replacement restorations had been placed in the remaining dentition and no further teeth had been lost. The patient however had remained under dental care of her GDP and had been diagnosed as suffering from Sjogrens syndrome, which had been confirmed by immunological investigations and a labial gland biopsy 6 years ago.

Intra-oral examination revealed an Angle's Class II division II malocclusion with a deep complete but non-traumatic overbite. The following teeth were present: UR76532(17, 16, 15, 13, 12), UL23457 (22, $23,24,25,27)$ and although heavily restored and stained were clinically and radiographically sound (Fig. 1). Although the patient's mouth was generally dry the oral hygiene was good and no active caries or periodontal disease was present.

At the time of the appointment she had been wearing her sectional denture for 14 years. The denture was still retentive and stable but the matrix acrylic resin and denture teeth had become stained and worn and an acrylic tooth had been lost from the UL6(26) region (Fig. 2).

\section{Comment}

Since this case had been restored many years ago, it was considered appropriate to consider more current options in treatment. In this respect the replacement of two upper central incisor teeth can be either by a fixed or removable prosthesis. In terms of fixed replacement the various options which could be considered are fixed bridgework, adhesive bridgework, spring-cantilevered bridgework or dental implants. For fixed bridgework to be successful however there must be adequate crown size and root support in the abutment teeth. In this case the 


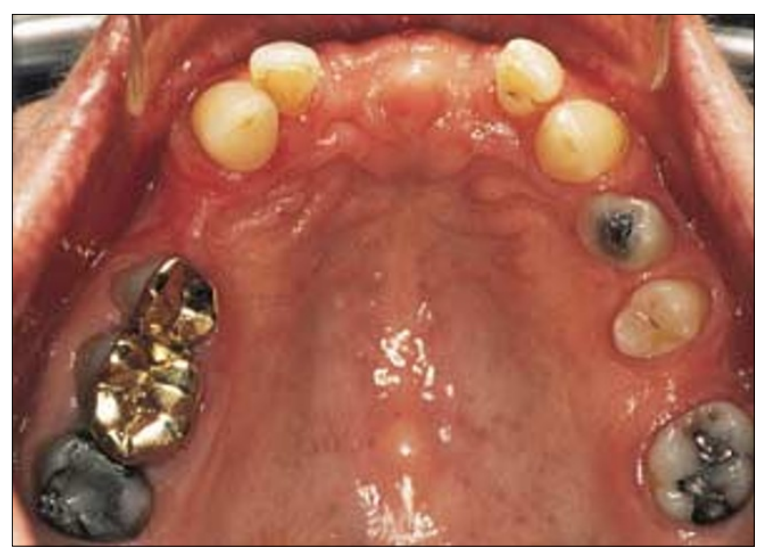

Fig. I An occlusal view of the maxillary arch

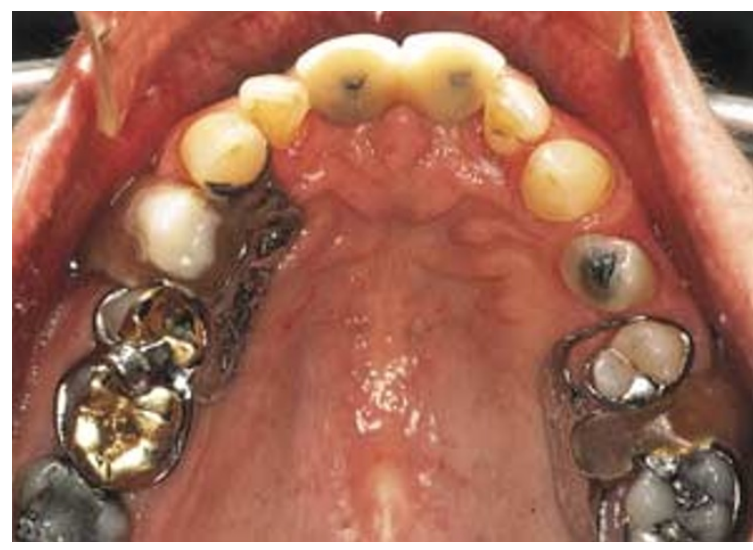

Fig. 2 An occlusal view with the original denture in place showing the wear and discoloration present remaining lateral incisors did not offer such support which would have necessitated involving the canine teeth. This would have resulted in elective crown destruction of four virgin teeth with the possibility of changed appearance, future pulp death and aesthetic problems with ongoing gingival recession. Adhesive bridgework, in the form of two cantilevered units, would also have been potentially hazardous with the small crowns of the lateral incisors being used for retention. In addition the deep complete overbite would have necessitated that the retaining wings would have carried the protrusive guidance and therefore would have had to be finished at the incisal level which would have compromised the aesthetic result. Spring cantilever bridgework from posterior units would be impossible as a result of the deep complete overbite. This would have meant that the bar connector could not have joined the abutment teeth to the prosthetic unit without affecting the occlusion. As far as dental implants were concerned these would have been technically possible, as there was adequate height and width of bone in the edentulous region. However the angulation of a subsequent crown to any implant would have been severe as a result of the retroclination of her remaining anterior teeth and the residual ridge in this region. In addition, the patient was not keen on surgery and was therefore not interested in such an option although this would not have been precluded by her age.

The options available as regards a removable prosthesis are an acrylic resin partial denture, a cobalt-chromium alloy partial denture, or some form of sectional or precision retained denture. For an acrylic resin partial denture to be successful an increase in the occlusal face height would be required to allow for space for the acrylic palate. This would have required a posterior onlay to be placed to restore the loss of contact of the posterior teeth as a result of the increase in vertical dimension. It had been determined at the original assessment 14 years previously that the patient could not tolerate any reduction in her freeway space and this was still the case. A cobalt chromium alloy denture would also require some form of connector to link the saddle to the tooth borne and retentive elements. Although cobaltchromium alloy can be made in a thin section, the complete overbite meant that no form of palatal coverage could be tolerated

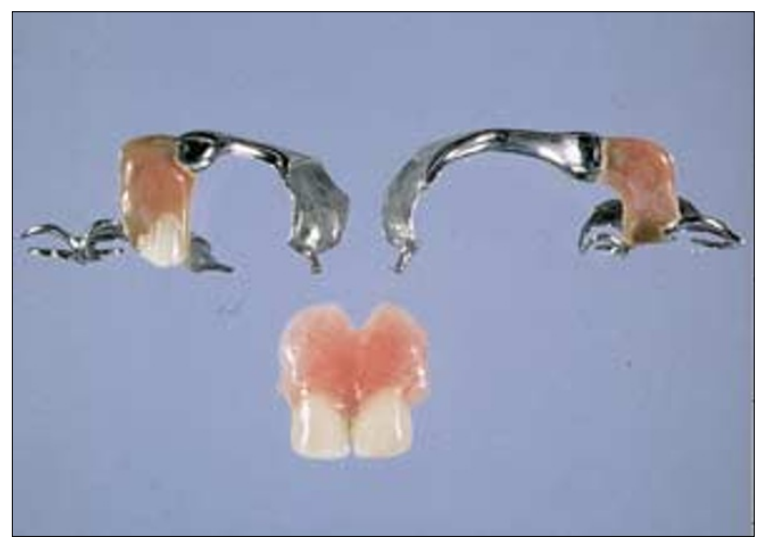

Fig. 3 An exploded view of the original three part sectional denture

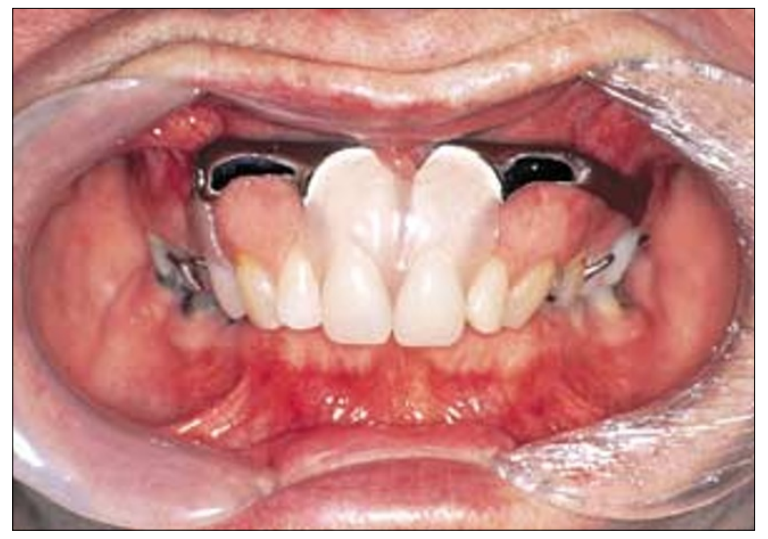

Fig. 4 Anterior view with the teeth in occlusion and the new denture in place 


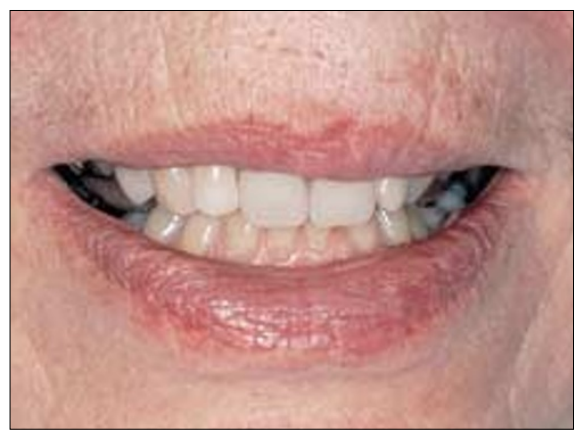

Fig. 5 The final aesthetic result

and therefore the only form of connector possible would involve a buccal connector. The severity of the undercut in the buccal sulcus region meant that a one-piece denture could not be placed. This left the only possible choice as being a sectional denture or the use of attachments connected to the neighbouring abutment teeth. The use of precision attachments would have involved preparation of these abutment teeth, which the patient was keen to avoid if possible. This left the use of a sectional denture as the only realistic option available.

\section{Treatment}

After careful analysis and surveying of the study casts it became apparent that the only design possible which would provide both an acceptable aesthetic and functional result was the three-part bilateral swinglock denture which had been the design of choice
14 years previously (Fig. 3). The old cobaltchromium alloy skeleton although functional, now exhibited signs of ageing particularly in relation to the hinges and split pins.

Master impressions were recorded therefore using silicone impression material and a hardened die cast was prepared and duplicated for the cobalt chromium base to be made. The resultant casting was tried in and after fine adjustments the matrix acrylic resin was added and the denture inserted (Fig. 4). The two posterior sections could be seated on their supporting abutment teeth and the separate swinglock arms rotated into position in the buccal sulcus. These arms were then locked in place by the placement of the anterior saddle, which had tubing that frictiongripped the split posts. In this anterior region the cobalt chromium alloy portion was masked using opaquer to eliminate the metal shining through the acrylic resin split pin section, although clearly it was not possible to eliminate this totally. The patient was delighted with the finished result (Fig. 5) and has now been wearing the replacement partial upper denture for 18 months.

\section{Conclusion}

The management of the edentulous space using complex partial denture designs such as split pins or swinglock dentures does not involve any special consideration in the clin- ical preparation; however an accurate silicone master impression is essential and the technical stages involve cobalt-chromium castings to be made to the highest specification. The technical expertise and support required in such cases is often difficult to find. The maintenance and recall of such dentures should follow standard denture procedure in that the patient should be seen for routine oral hygiene and denture hygiene procedures as required by the individual's needs and the denture should be reviewed six-monthly regarding any activation of pins or locking mechanisms. This unusual case shows that with the correct treatment planning and management a case such as this, even by the use of a complex partial denture framework, can be extremely successful in terms of life expectancy and if maintained properly does not endanger the remaining dentition in any way.

1 Pullen-Warner E, L'Estrange P R. Sectional dentures: a clinical and technical manual. New York: Wright and Sons, 1978: 18.

2 Lee J H. Sectional partial metal dentures incorporating an internal locking bolt. $J$ Prosthet Dent 1963; 13: 1067-1075.

3 Dummer P M H, Gidden J. The upper anterior sectional denture. J Prosthet Dent 1979; 41: 146-152.

4 Simmons J J. Swinglock stabilization and retention. A preliminary clinical report. Tex Dent J 1963; 81: 10-12.

5 Russell M D, Turner P. A three-part sectional design for an upper removable partial denture with an anterior modification. Br Dent J 1987; 162: 24-26. 